\title{
Association of leg length to measures of body fatness in British children aged 5-15 years
}

\author{
Triantafyllos Pliakas ${ }^{1}$ and H. David McCarthy ${ }^{2}$ \\ ${ }^{1}$ Department of Public Health and Policy, London School of Hygiene and Tropical Medicine, London, UK and ${ }^{2}$ London \\ Metropolitan University, London, $U K$
}

Both leg length (LL) and childhood obesity independently impact on adult health. One study in children has found an inverse relationship between relative LL and higher body fatness (BF), using skinfold measurements ${ }^{(1)}$. Moreover, adult studies suggest that relative shorter $\mathrm{LL}$ is associated with increased $\mathrm{BF}^{(2)}$. The present cross-sectional study has examined measures of LL to BF in British children.

A secondary analysis of previously collected anthropometric data ${ }^{(3)}$ was carried out in children aged 5-15 years ( $n$ 7721; males 3411). Outcome measures included height, BMI, waist circumference (WC) and LL expressed as standard deviation scores (SDS) using current references. LL was directly measured to the nearest $0.1 \mathrm{~cm}$. Relative LL was defined as LL: height (LLHR). Data for boys and girls were statistically analysed separately in 1-year intervals. Overweight and obesity was defined as BMI SDS >1.33 (total fatness) or WC SDS $>1.33$ (central fatness), both of which correspond to the 91st centile. A Mann-Whitney test was used to assess the differences in LLHR between non-overweight children and overweight and obese children.

Overweight and obese children tended to be taller with longer legs, especially when WC was used to define overweight and obesity. However, these children had a relatively shorter LL across most ages. In particular, overweight and obese boys aged 11-13 years had a significantly lower LLHR than non-overweight boys $(P=0.008-P=0.001$ for BMI; $P=0.002-P=0.006$ for WC). Furthermore, overweight and obese boys (based on BMI) had a significantly lower LLHR at ages 5, 7, 10 and 14 years $(P<0.05$ for all comparisons). Overweight and obese boys had a higher LLHR only at ages 6 and 9 years when WC was used, and at ages 9 and 15 years when BMI was used ( $P>0.05$ for all comparisons). In girls lower LLHR was more closely related to total fatness rather than central fatness. Using BMI overweight and obese girls had a significantly lower LLHR at ages 5, 10, 11, 13, 14 and 15 years $(P=0.021-P=0.002)$. Using WC a tendency towards a lower LLHR was observed in overweight and obese girls at all ages except at 8 years $(P>0.05$ for all comparisons).
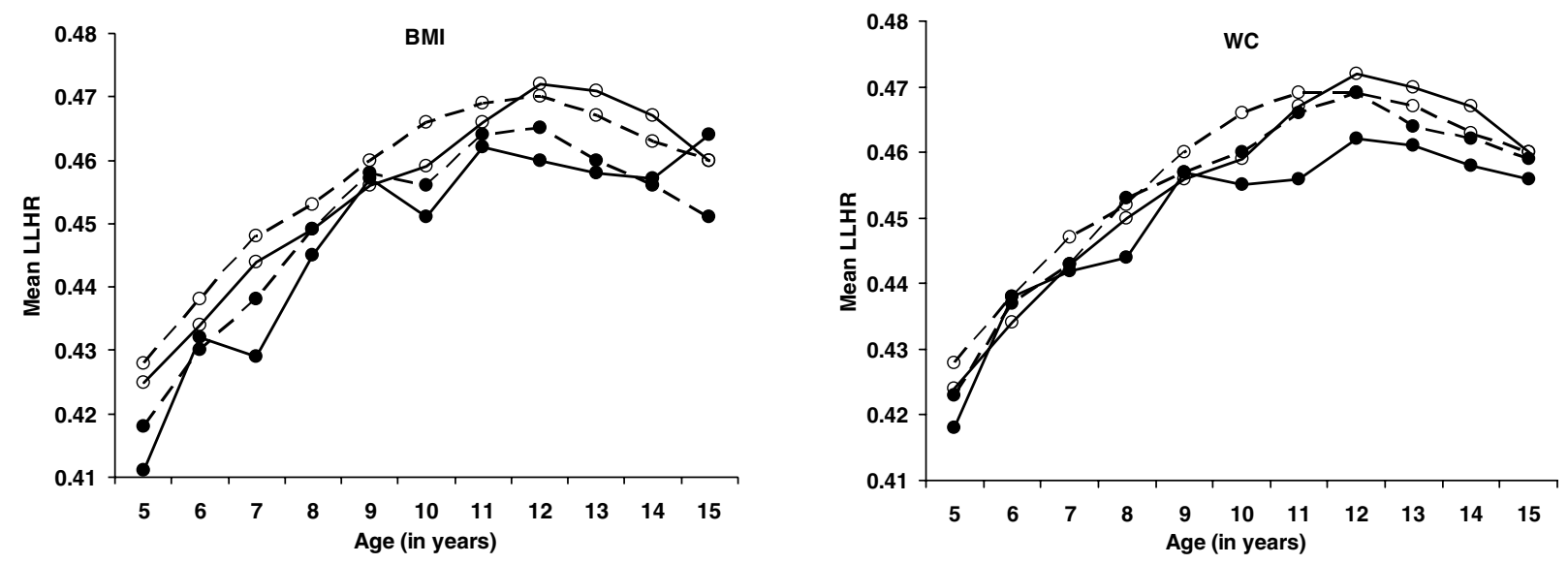

Figure. Mean LLHR by age in non-overweight $(\bigcirc)$ and overweight and obese $(\mathbf{O})$ boys $(-)$ and girls $(--)$ aged 5-15 years

The present study indicates that children with relatively shorter legs tend to be fatter based on either BMI or WC as the measure of fatness. However, the evidence is weaker when using WC, particularly in girls. Theoretically, this outcome is logical as LL is a component of the denominator of BMI. These findings would also suggest that trunk length must be relatively greater in overweight and obese children. Whilst disproportionate body dimensions appear to be linked to measures of BF in children, longitudinal studies are needed to confirm this suggestion.

1. Frisancho AR (2007) Am J Hum Biol 19, 703-710.

2. Asao K, Kao WH, Baptiste-Roberts K, Bandeen-Roche K, Erlinger TP \& Brancati FL (2006) Diabetes Care 29, 1632-1637.

3. British Standards Institute (1990) Body Measurements of Boys and Girls from Birth up to 16.9 Years, BS7231. London: British Standards Institute. 